\title{
Procalcitonin in the context of delayed cerebral ischemia after aneurysmal subarachnoid hemorrhage
}

\author{
Michael Veldeman, MD,' Daniel Lepore, MD,,3 Anke Höllig, MD, ${ }^{2}$ Hans Clusmann, MD, ${ }^{1}$ \\ Christian Stoppe, MD, ${ }^{2}$ Gerrit Alexander Schubert, MD, ${ }^{1}$ and Walid Albanna, MD${ }^{1}$

\begin{abstract}
Departments of ${ }^{1}$ Neurosurgery and ${ }^{2}$ Intensive Care and Intermediate Care, Rheinisch-Westfälische Technische Hochschule (RWTH) Aachen University, Aachen, Germany; and ${ }^{3}$ Department of Anesthesia and Intensive Care Medicine, Centre Hospitalier
\end{abstract} \\ Universitaire de Liège, Belgium
}

\begin{abstract}
OBJECTIVE Aneurysmal subarachnoid hemorrhage (aSAH) initiates a deleterious cascade activating multiple inflammatory processes, which can contribute to delayed cerebral ischemia (DCl). Procalcitonin (PCT) is an established marker for sepsis treatment monitoring, and its time course in the context of $\mathrm{DCl}$ after aSAH remains unclear. The aim of this trial was to assess the predictive and confirmative value of PCT levels in the context of DCl.

METHODS All patients admitted to the authors' institution with aSAH between 2014 and 2018 were prospectively screened for eligibility. Daily PCT levels were recorded alongside relevant aSAH characteristics. The predictive and confirmative values of PCT levels were assessed using a receiver operating characteristic and area under the curve (AUC) analysis. The course of PCT levels around the DCl event was evaluated in an infection-free subgroup of patients.

RESULTS A total of 132 patients with aSAH were included. Early PCT levels (first 3 days post-aSAH) had a low predictive value for the development of DCI (AUC 0.661, standard error [SE] 0.050; $p=0.003$ ) and unfavorable long-term outcome (i.e., Glasgow Outcome Scale-Extended scores 1-4; AUC 0.674, SE 0.054; $p=0.003$ ). In a subgroup analysis of infection-free patients $(n=72)$, PCT levels were higher in patients developing $\mathrm{DCl}(p=0.001)$ and DCl-related cerebral infarction ( $p=0.002$ ). PCT concentrations increased gradually after $\mathrm{DCl}$ and decreased with successful intervention. In refractory cases progressing to cerebral infarction, PCT levels showed a secondary increase.

CONCLUSIONS Early higher PCT levels were associated with the later development of DCI and unfavorable outcome. Analysis of PCT beyond the first couple of days after hemorrhage is hampered by nosocomial infections. In infection-free patients, however, PCT levels rise during $\mathrm{DCl}$ and an additional increase develops in patients developing cerebral infarction.

Clinical trial registration no.: NCT02142166 (clinicaltrials.gov)

https://thejns.org/doi/abs/10.3171/2020.5.JNS201337
\end{abstract}

KEYWORDS subarachnoid hemorrhage; delayed cerebral ischemia; procalcitonin; cerebral aneurysm; vascular disorders

$\mathrm{D}$ ESPITE general advancement in intensive care treatment, the outcome after aneurysmal subarachnoid hemorrhage (aSAH) remains poor., ${ }^{1,2}$ Following early mortality, delayed cerebral ischemia (DCI) is an important contributing factor to additional morbidity and mortality. ${ }^{3}$ DCI affects approximately $20 \%-30 \%$ of survivors of aSAH and typically occurs between days 4 and 14 after the initial hemorrhage. The concept of angiographic vasospasm as the sole culprit of delayed cerebral infarctions has been largely abandoned, and clinical as well as experimental research indicates a considerably more complex nature of the underlying pathophysiology. ${ }^{4} \mathrm{Re}-$ cent studies substantiated that cerebral vasospasm is only a contributing factor to DCI, alongside microcirculatory spasms, microthrombi formation, cortical spreading depolarization, and disruption of cerebral autoregulation. ${ }^{4-7}$ The initial increase of intracranial pressure and concomitant reduction of cerebral perfusion pressure initiate a deleterious cascade involving cerebral damage and inflammation. A term for this concept has been coined: early brain injury (EBI). ${ }^{8}$ Subsequently, in response to the subarachnoid extravascular blood, leukocyte infiltration takes

ABBREVIATIONS aSAH = aneurysmal subarachnoid hemorrhage; $\mathrm{AUC}=$ area under the curve; $\mathrm{CRP}=\mathrm{C}$-reactive protein; $\mathrm{DCl}=$ delayed cerebral ischemia; $\mathrm{EBI}=$ early brain injury; GOSE = Glasgow Outcome Scale-Extended; $\mathrm{HH}=$ Hunt and Hess; IL = interleukin; noDCl = subgroup of aSAH patients without DCl; PCT = procalcitonin; ROC = receiver operating characteristic; SE = standard error; SIRS = systemic inflammatory response syndrome; UTI = urinary tract infection.

SUBMITTED April 18, 2020. ACCEPTED May 18, 2020.

INCLUDE WHEN CITING Published online September 4, 2020; DOI: 10.3171/2020.5.JNS201337. 
place, resulting in the release of inflammatory cytokines. Additional microglia activation and macrophage diapedesis contribute to the activation of a widespread inflammatory cascade. ${ }^{9,10}$ The resulting activation of a systemic immune response after aSAH is reflected by increased levels of circulating cytokines such as interleukin (IL)-1, IL-6, and tumor necrosis factor $-\alpha .{ }^{9}$

Leukocyte count appears to correlate best with DCI occurrence and with a higher risk for symptomatic vasospasm and DCI. ${ }^{11}$ Conflicting results exist concerning the levels of C-reactive protein (CRP) after aSAH. In a prospective trial, CRP levels correlated with outcome but did not predict DCI or infarction. ${ }^{12}$ However, in a post hoc analysis of Simvastatin in Aneurysmal Subarachnoid Hemorrhage (i.e., STASH) study data, CRP levels were predictive of secondary deterioration in patients with good-grade aSAH. ${ }^{13}$ High levels of IL-6 in serum as well as spinal fluid have been linked to the occurrence of DCI and poor outcome. ${ }^{14,15}$ In contrast to other acute-phase proteins, which are produced by either leukocytes or endothelial cells (e.g., IL-6, tumor necrosis factor- $\alpha$, and interferon- $\alpha$ ) or in the liver (CRP), procalcitonin (PCT) is synthesized by parafollicular cells of the thyroid and by neuroendocrine cells of the lung and the intestine. These expression sites result in different biodynamic properties opposed to the aforementioned conventional inflammatory markers, making PCT an interesting target to examine in patients with aSAH.

Apart from bacterial infections, other conditions can also induce an increase in PCT levels. An early and transient release of PCT into the circulation was observed after severe trauma, and the amount of circulating PCT was proportional to the severity of tissue injury and hypovolemia. ${ }^{16}$

The aim of this study was to assess the predictive and confirmative value of PCT levels in the context of DCI up to 21 days after aSAH. In order to improve consistency in reporting, we tried to adhere as much as possible to standing recommendations for reporting data on biomarkers in aSAH. ${ }^{17}$

\section{Methods}

\section{Patient Population and Study Design}

This prospective observational study, performed at RWTH Aachen University Hospital, was approved by the local ethics committee and was registered with the ClinicalTrials.gov database (registration no. NCT02142166). All patients admitted to our institution between 2014 and 2018 with confirmed aSAH who were between 18 and 90 years of age were screened for eligibility. Informed consent was obtained from the patient or his or her next of kin. Clinical severity at the time of admission was assessed by the on-call neurosurgeon according to the Hunt and Hess (HH) grading scale, and radiological severity was assessed using the modified Fisher scale by a neuroradiologist. Patients were excluded in the following cases: age younger than 18 years; pregnancy; lack of signed informed consent; taking a study drug within the last 30 days; or simultaneous participation in another clinical trial (except participation in a control group). Patients with infectious complications were identified according to standing systemic inflammatory response syndrome (SIRS) and sepsis criteria ${ }^{18}$ and removed from specific subgroup analyses. Pneumonia was defined as a new or progressive pulmonary infiltrate on chest radiographs with positive culture swabs. A catheter-related infection was considered in the case of positive blood cultures, with confirmation by catheter tip cultures; a urinary tract infection (UTI) was defined as $\geq 10^{5}$ colony-forming units, or as positive urinalysis (defined as positive leukocyte esterase or nitrate or $>5$ leukocytes $/ \mathrm{ml}$ ) and $\geq 1000$ colony-forming units. Meningitis was defined by positive CSF bacterial cultures from a ventriculostomy drain or lumbar puncture. Evaluation for infections was done daily by our intensivist staff and documented rigorously in the medical records.

\section{Standard Treatment Algorithm}

All patients were treated according to a standardized treatment protocol as previously published. ${ }^{19,20}$ In brief, after diagnosis of the aSAH, aneurysms were secured within 48 hours via surgical clipping or endovascular coiling. All patients were monitored at our neurointensive care unit. In accordance with the treatment protocol, all patients received prophylactic enteral nimodipine. ${ }^{21}$

After failing an initial wake-up test, patients with a persistent state of reduced consciousness were considered for placement of invasive neuromonitoring as recommended by the 2014 consensus statement. 22,23 The occurrence of DCI was determined by clinical ${ }^{24}$ or by functional deterioration as documented by a new CT perfusion deficit (cerebral blood flow/mean transit time mismatch with a characterizing territorial or watershed distribution) or an oxygenation (brain tissue oxygen pressure $\left[\mathrm{p}_{\mathrm{ti}} \mathrm{O}_{2}\right]<10 \mathrm{~mm}$ $\mathrm{Hg}$ ) or metabolic (lactate/pyruvate ratio $\geq 40$ ) crisis as measured by intraparenchymal probes. Before confirming the diagnosis, other causes (i.e., untreated hydrocephalus, rebleeding, infection, electrolyte imbalance, or seizure) were ruled out. After diagnosis of DCI, first-tier treatment was started, consisting of induced euvolemic arterial hypertension ( $\geq 180 \mathrm{~mm} \mathrm{Hg}$ ) by means of intravenous noradrenaline infusion. If refractory to induced hypertension, patients were considered for second-tier endovascular rescue treatment by either transluminal balloon angioplasty or intraarterial spasmolysis, depending on the localization and distribution of spastic vessels. ${ }^{20}$ Cerebral infarctions diagnosed during ongoing DCI or as a first sign of DCI confirmed by at least two modalities were called DCI-related infarction. Embolic stroke due to halted anticoagulation in patients with cardiac arrhythmias or as a result of endovascular aneurysm treatment was identified and excluded based on history and presentation on imaging.

\section{Sampling and Data Collection}

Systemic PCT levels (in $\mu \mathrm{g} / \mathrm{L}$ ) were collected once daily (6:00 AM) during routine arterial blood sampling into a standard serum tube, starting on admission until discharge from the ICU. This resulted in one PCT value per day for each patient. Samples were directly transferred to our institutional laboratory and centrifuged. All analyses were performed in accordance with institutional labora- 
tory standards and internal quality control. Blood sampling and measurements were part of the clinical routine and no additional sampling was performed. We assessed and documented the occurrence of SIRS, sepsis, catheter infection, pneumonia, meningitis, and UTIs as part of the routinely used ICU electronic health records. Organ dysfunction was assessed using the Sequential Organ Failure Assessment (SOFA) score. ${ }^{25}$ All analyses are based on a single daily PCT value per patient. The occurrence of DCI and DCI-related cerebral infarction was recorded, and clinical outcome according to the Glasgow Outcome Scale-Extended (GOSE) was assessed after 12 months by analysis of patient files or a structured telephone interview by a trained independent investigator working in blinded fashion. ${ }^{26}$

\section{Subgroup Comparison}

A predefined subgroup comparison was planned in which patients were dichotomized according to good or poor clinical grade $\left(\mathrm{HH}_{\mathrm{I}-\mathrm{II}} \mathrm{Vs} \mathrm{HH}_{\mathrm{III}-\mathrm{V}}\right)$, presence or absence of DCI (DCI vs noDCI), DCI with or without cerebral infarction $\left(\mathrm{DCI}_{\text {infarct }} \mathrm{vS} \mathrm{DCI}_{\text {no-infarct }}\right)$, and favorable or unfavorable outcome after 12 months $\left(\mathrm{GOSE}_{5-8}\right.$ vs $\left.\mathrm{GOSE}_{1-4}\right)$. For every subgroup a representative time frame was identified and values per patient were averaged over this period. For the comparison of dichotomized clinical grading $\left(\mathrm{HH}_{\mathrm{I}-\mathrm{II}}\right.$ vs $\left.\mathrm{HH}_{\mathrm{III}-\mathrm{V}}\right)$ and dichotomized clinical outcome $\left(\mathrm{GOSE}_{5-8}\right.$ vs GOSE $\left._{1-4}\right)$, the first available PCT levels $\left(\mathrm{d}_{0-2}\right)$ were used in order to evaluate a predictive effect. For comparison of DCI and noDCI subgroups, PCT levels on the day of DCI occurrence and the 2 following days were averaged to evaluate a confirmative effect. In the noDCI group, values from days 6 to 8 were averaged, with day 6 being the average time point of DCI occurrence in this cohort. Comparing infarction and no-infarction subgroups, averaged PCT data of days 6-8 after DCI development were analyzed. Early PCT was defined as the first available PCT level after ictus.

\section{Statistical Analysis}

All data are presented as mean and standard deviation for normally distributed and as median and interquartile range (IQR) for nonnormally distributed continuous variables. Categorical variables are depicted as frequencies and proportions. After normality testing using plotting and the Shapiro-Wilk test, the appropriate statistical test was selected. For normally distributed continuous data the unpaired t-test was used, and for nonnormally distributed data the Mann-Whitney U-test was used. Curves of PCT values were compared within and between groups by a repeated-measures ANOVA with a Greenhouse-Geisser correction for sphericity. Due to the natural skewness of PCT data, absolute values were logarithmically transformed before plotting in the subgroup comparisons. The predictive value of early PCT was assessed using a receiver operating characteristic (ROC) and area under the curve (AUC) analysis. Cutoff values were calculated by means of the Youden index in order to estimate diagnostic sensitivity and specificity as well as positive and negative predictive values. Predictors of unfavorable out- come were further tested in a binomial logistic regression model. Continuous covariates were tested for linearity using a Box-Tidwell procedure. Predictor covariates were included when a $\mathrm{p}$ value $<0.1$ was identified in univariate testing.

Missing outcome data were not imputed. All statistical analyses were performed using IBM SPSS Statistics version 25 (IBM Corp.). Statistical significance was defined as a 2 -sided $p<0.05$. Graphics were plotted using GraphPad Prism version 8.1.1.

\section{Results \\ Baseline Characteristics}

During the 4-year inclusion period, 169 consecutive patients with aSAH were prospectively assessed for eligibility. A total of 37 patients had to be excluded based on incomplete PCT measurements. For these patients, PCT measurement was temporarily stopped due to technical laboratory problems. In Supplementary Table 1 we compared included patients versus patients excluded due to missing PCT data. Because these values were missing at random, the group of missing cases proved representative according to all relevant baseline characteristics, reducing the chance of attrition bias. The mean age of the remaining 132 patients was $55.9 \pm 12.0$ years (range $23-90$ years), and the female/male ratio was $91(68.9 \%) / 41(31.1 \%)$. A total of $63(47.7 \%)$ patients were invasively monitored, among whom were 21 (15.9\%) good-grade patients. Sixty-four (48.5\%) patients developed DCI and, of those, 32 (24.2\%) progressed to DCI-related cerebral infarctions. A total of $60(45.5 \%)$ patients developed relevant bacterial infection during their ICU stay. The recruitment process is depicted in Fig. 1. Favorable outcome after 12 months was attained in $78(64.5 \%)$ patients, with $11(8.3 \%)$ overall cases lost to follow-up. All relevant baseline characteristics are depicted in Table 1.

\section{The Course of PCT After aSAH}

The time course of PCT (in $\mu \mathrm{g} / \mathrm{L}$ ) in patients with aSAH who had infection $(n=60)$ was plotted in comparison to the infection-free group $(n=72)$ (Fig. 2). In both groups an initial stable baseline of PCT levels was visible.

In the infection-free subgroup, PCT levels remained stable over time as confirmed by a 1-way repeated-measures ANOVA with a Greenhouse-Geisser correction $(F[4.874,34.120]=0.626, \mathrm{p}=0.677)$. In the subgroup of patients with infection, 3 PCT peaks compared with PCT baseline levels $\left(\mathrm{d}_{0-2}\right)$ were discernible: day $8(1.72$, IQR $0.27 \mu \mathrm{g} / \mathrm{L} ; \mathrm{p}=0.035)$, day $13(1.73$, IQR $0.20 \mu \mathrm{g} / \mathrm{L}$; $\mathrm{p}=0.049)$, and day $16(1.68$, IQR $0.12 \mu \mathrm{g} / \mathrm{L} ; \mathrm{p}=0.179)$. Analyzing electronic patient records in more detail, these peaks were associated with the development of SIRS ( $\mathrm{n}=$ $16)$, sepsis $(\mathrm{n}=24)$, pneumonia $(\mathrm{n}=16), \mathrm{UTI}(\mathrm{n}=11)$, and/ or meningitis $(n=16)$ during the ICU stay.

\section{Early PCT Levels and DCI}

Because early PCT levels $\left(\mathrm{d}_{0-2}\right)$ did not differ significantly between patients who remained infection free and those developing nosocomial infections later (0.091, IQR $0.104 \mu \mathrm{g} / \mathrm{L}$ vs 0.133 , IQR $0.095 \mu \mathrm{g} / \mathrm{L} ; \mathrm{p}=0.089)$, data 
TABLE 1. Baseline characteristics of the whole cohort of patients with aSAH and of the infection-free subgroup

\begin{tabular}{|c|c|c|c|c|}
\hline Characteristic & $\begin{array}{l}\text { All Patients, } \\
n=132\end{array}$ & $\begin{array}{c}\text { Infection, } \\
n=60\end{array}$ & $\begin{array}{c}\text { Infection Free, } \\
n=72\end{array}$ & $\begin{array}{c}p \\
\text { Value }\end{array}$ \\
\hline Age in yrs, mean \pm SD (range) & $55.9 \pm 12.0(23-90)$ & $58.1 \pm 12.5(32-90)$ & $54.3 \pm 11.3(23-83)$ & 0.093 \\
\hline \multicolumn{4}{|l|}{ Sex } & \multirow[t]{3}{*}{0.241} \\
\hline Female & $91(68.9 \%)$ & $39(65.0 \%)$ & $52(72.2 \%)$ & \\
\hline Male & $41(31.1 \%)$ & $21(35.0 \%)$ & $20(27.8 \%)$ & \\
\hline \multicolumn{4}{|l|}{ Aneurysm location } & \multirow[t]{3}{*}{0.171} \\
\hline Anterior circulation & $104(78.8 \%)$ & $50(83.3 \%)$ & $54(75.0 \%)$ & \\
\hline Posterior circulation & $28(21.2 \%)$ & $10(16.7 \%)$ & $18(25.0 \%)$ & \\
\hline Clipping & $60(45.5 \%)$ & $27(45.0 \%)$ & $32(44.4 \%)$ & \multirow{2}{*}{0.539} \\
\hline Coiling & $72(54.5 \%)$ & $32(55.0 \%)$ & $39(55.6 \%)$ & \\
\hline \multicolumn{4}{|l|}{$\mathrm{HH}$ grade } & \multirow{2}{*}{$<0.001$} \\
\hline Grade I & $25(18.9 \%)$ & $6(10.0 \%)$ & $19(26.4 \%)$ & \\
\hline Grade II & $32(24.2 \%)$ & $10(16.7 \%)$ & $22(30.6 \%)$ & \\
\hline Grade III & $44(33.3 \%)$ & $19(31.7 \%)$ & $25(34.7 \%)$ & \\
\hline Grade IV & $17(12.9 \%)$ & $13(21.7 \%)$ & $4(5.6 \%)$ & \\
\hline Grade V & $14(10.6 \%)$ & $12(20.0 \%)$ & $2(2.8 \%)$ & \\
\hline \multicolumn{5}{|l|}{ ICU parameter } \\
\hline LOS in days, mean \pm SD & $26.2 \pm 16.7$ & $35.4 \pm 18.4$ & $18.4 \pm 9.9$ & $<0.001$ \\
\hline Mechanical ventilation & $76(57.6 \%)$ & $56(93.3 \%)$ & $6(8.3 \%)$ & $<0.001$ \\
\hline $\begin{array}{l}\text { Duration of ventilation in } \\
\text { days, mean } \pm \text { SD }\end{array}$ & $23.3 \pm 17.8$ & $26.6 \pm 18.2$ & $14.8 \pm 13.8$ & 0.006 \\
\hline Tracheostomy & $49(37.1 \%)$ & $40(66.7 \%)$ & $9(12.5 \%)$ & $<0.001$ \\
\hline \multicolumn{4}{|l|}{ Modified Fisher grade } & \multirow[t]{2}{*}{0.001} \\
\hline Grade 1 & $34(25.8 \%)$ & $6(10.0 \%)$ & $28(38.9 \%)$ & \\
\hline Grade 2 & $18(13.6 \%)$ & $7(11.7 \%)$ & $11(15.3 \%)$ & \\
\hline Grade 3 & $30(22.7 \%)$ & $19(31.7 \%)$ & $11(15.3 \%)$ & \\
\hline Grade 4 & $50(37.9 \%)$ & $28(46.7 \%)$ & $22(30.6 \%)$ & \\
\hline \multicolumn{4}{|l|}{ Clinical outcome* } & \multirow[t]{4}{*}{$<0.001$} \\
\hline Favorable, GOSE ${ }_{5-8}$ & $78(64.5 \%)$ & $17(34.7 \%)$ & $61(84.7 \%)$ & \\
\hline Unfavorable, GOSE ${ }_{1-4}$ & $43(35.5 \%)$ & $32(65.3 \%)$ & $11(15.3 \%)$ & \\
\hline Mortality & $24(18.2 \%)$ & $20(33.3 \%)$ & $4(5.6 \%)$ & \\
\hline \multicolumn{5}{|l|}{$\mathrm{DCl}$} \\
\hline $\mathrm{DCl}$ occurrence & $64(48.5 \%)$ & $41(68.3 \%)$ & $23(31.9 \%)$ & $<0.001$ \\
\hline Cerebral infarction & $32(24.2 \%)$ & $25(41.7 \%)$ & $7(9.7 \%)$ & $<0.001$ \\
\hline \multicolumn{5}{|l|}{ Infections } \\
\hline SIRS & $59(44.7 \%)$ & $59(98.3 \%)$ & NA & NA \\
\hline Sepsis & $24(18.2 \%)$ & $24(40.0 \%)$ & NA & NA \\
\hline Pneumonia & $52(39.4 \%)$ & $52(86.7 \%)$ & NA & NA \\
\hline UTI & $18(13.6 \%)$ & $18(30.0 \%)$ & NA & NA \\
\hline Meningitis & $16(12.1 \%)$ & $16(26.7 \%)$ & NA & NA \\
\hline Total & $60(45.5 \%)$ & $60(100 \%)$ & NA & NA \\
\hline \multicolumn{5}{|c|}{$\begin{array}{l}\text { LOS = length of hospital stay; NA = not applicable. } \\
\text { Unless otherwise indicated, values are expressed as the number of patients (\%). Boldface type indicates statistical } \\
\text { significance. Due to the exclusion of patients developing nosocomial infections, a selection bias in favor of patients with } \\
\text { less severe aSAH, based on the HH and modified Fisher scales, is introduced. This is reflected by the shorter length of } \\
\text { ICU stay and lower proportion of patients receiving ventilation in the infection-free subgroup. } \\
\text { * Outcome is missing in } 11 \text { patients in the infection group. }\end{array}$} \\
\hline
\end{tabular}




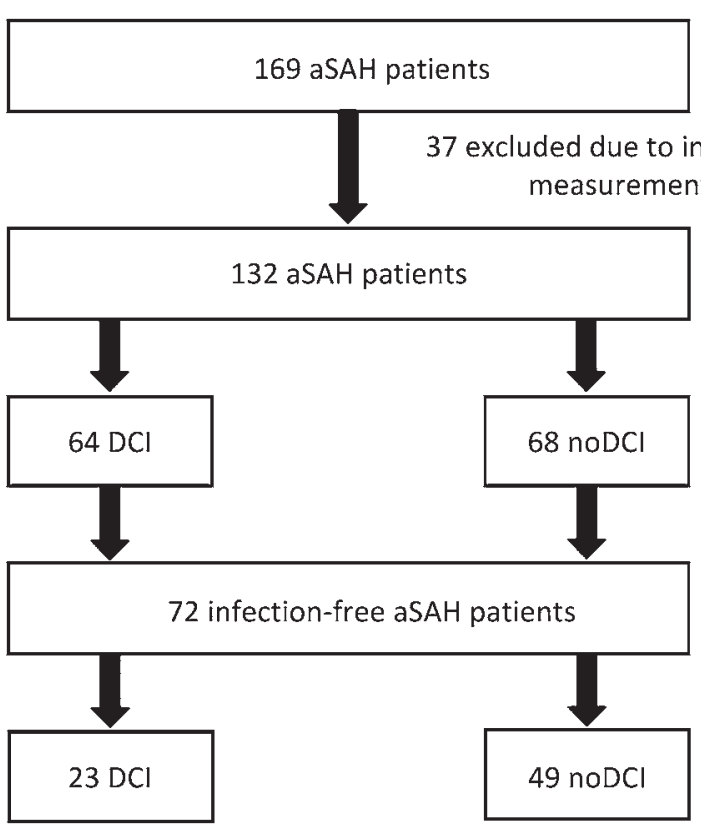

FIG. 1. Flowchart of patient recruitment. A total of 37 patients were excluded due to insufficient measurements. In the final analysis, 132 patients with aSAH were included. For the analysis of the confirmative value of PCT levels on the occurrence of $\mathrm{DCl}$ and $\mathrm{DCl}$-related infarction, the analysis was restricted to infection-free patients $(n=72)$.

from all patients $(n=132)$ were used when evaluating the predictive value of early PCT $\left(\mathrm{d}_{0-2}\right)$ measurements.

We compared early PCT $\left(\mathrm{d}_{0-2}\right)$ in patients developing and not developing DCI. The median PCT of patients in whom DCI developed later was significantly higher compared with those who did not (DCI 0.125, IQR $0.215 \mu \mathrm{g} / \mathrm{L}$ vs noDCI 0.070 , IQR $0.080 \mu \mathrm{g} / \mathrm{L} ; \mathrm{p}=0.023$ ), as depicted in Fig. 3A. In the ROC analysis the AUC demonstrated a low predictive value in identifying patients who will develop DCI based on PCT (AUC 0.661, standard error [SE] 0.050; $p=0.003$ ) (Fig. 3B). Focusing on the infection-free subgroup alone, results proved comparable (AUC 0.665 , SE 0.069; $p=0.039$ ). The optimal diagnostic cutoff score for later DCI development was calculated at 0.086 $\mu \mathrm{g} / \mathrm{L}$ and was associated with a sensitivity of $62.7 \%$ and specificity of $62.1 \%$, corresponding to a positive predictive value of $61.02 \%(95 \%$ CI $51.77 \%-69.57 \%)$ and a negative predictive value of $60.34 \%$ (95\% CI 50.96\%-69.02\%).

The performance of PCT as a predictor of DCI occurrence in the ROC analysis was comparable to that of the modified Fisher scale (AUC 0.673, SE 0.071; $p=0.030$ ) and $\mathrm{HH}$ grading scale (AUC 0.638, SE 0.073; $\mathrm{p}=0.073$ ) in this cohort.

\section{Early PCT Levels and Outcome}

Patients developing unfavorable $\left(\mathrm{GOSE}_{1-4}\right)$ outcome presented with higher early PCT $\left(\mathrm{d}_{0-2}\right)$ compared with those who recovered with a favorable $\left(\mathrm{GOSE}_{5-8}\right)$ outcome (0.115, IQR $0.120 \mu \mathrm{g} / \mathrm{L}$ vs 0.060 , IQR $0.104 \mu \mathrm{g} / \mathrm{L} ; \mathrm{p}=$ 0.025) (Fig. 3C). Early PCT levels showed a low predictive value concerning outcome prediction (AUC 0.674, SE 0.054; $\mathrm{p}=0.003$ ) (Fig. 3D).

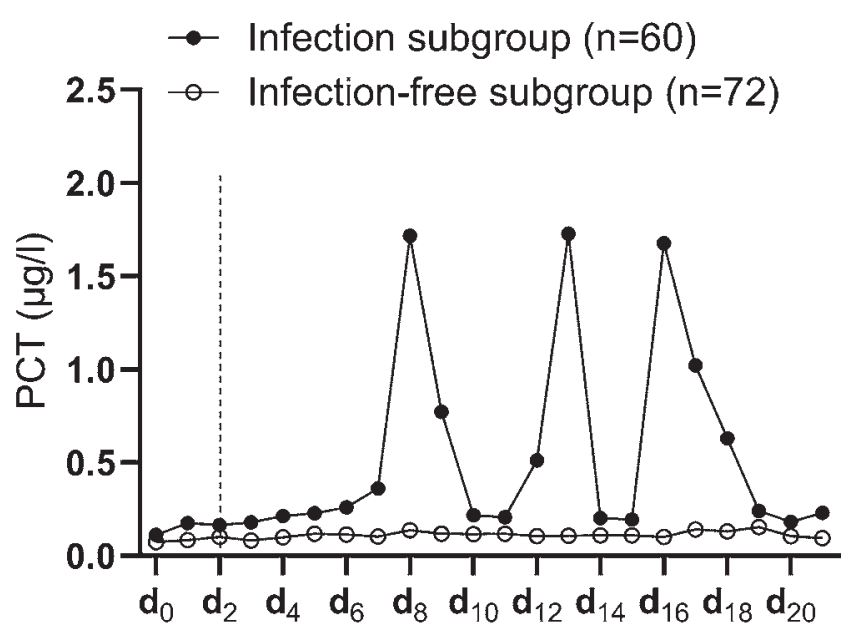

FIG. 2. Time course of median PCT levels (in $\mu \mathrm{g} / \mathrm{L}$ ) during the first 21 days after hemorrhage ( $d_{0}$ is the day of hemorrhage) in all patients with aSAH and infection $(n=60)$ and in all patients with aSAH without infection $(n=72)$. In both groups an initial plateau phase $\left(d_{0-2}\right)$ was not significant $(p=0.089)$. In the infection-free subgroup, cases with SIRS, sepsis, and other bacterial infections were excluded. In this group, PCT levels remained stable over time, as confirmed by a 2-way repeated-measures ANOVA with a Greenhouse-Geisser correction ( $F[4.874,34.120]=$ $0.626, p=0.677$ ). In the data of patients with infection, a peak in PCT levels develops on days 8,13 , and 17 of the ICU stay, corresponding to the development of SIRS $(n=16)$, sepsis $(n=24)$, pneumonia $(n=16)$, UTI $(n=11)$, and/or meningitis $(n=16)$ at these time points.

After additional univariate testing, $\mathrm{HH}(\mathrm{p}<0.001)$ and modified Fisher grading $(\mathrm{p}=0.071)$ were identified as covariates. A binomial logistic regression model was performed for all patients, with early PCT levels compared with $\mathrm{HH}$ and modified Fisher grading as covariates. The model was significant $\left(\chi^{2}(8)=29.098 ; \mathrm{p}<0.001\right)$ and explained $55.5 \%$ (Nagelkerke $\mathrm{R}^{2}$ ) of the variance in the occurrence of unfavorable outcome. Of the 3 predictor variables, only $\mathrm{HH}$ grading $(\mathrm{p}<0.001)$ and early PCT $(\mathrm{p}$ $=0.048$ ) were associated with an increased likelihood of developing an unfavorable outcome.

\section{PCT Levels Around the Primary DCI Event}

When comparing PCT data beyond day 2 , the analyses were restricted to the subgroup of infection-free patients $(n=72)$ to exclude the effect of nosocomial infections on PCT beyond this time point. In a comparison between the DCI $(\mathrm{n}=23)$ and noDCI $(\mathrm{n}=49)$ subgroups, PCT levels were higher in patients with DCI, indicating a confirmative value of PCT levels for DCI occurrence (DCI 0.163, IQR $0.179 \mu \mathrm{g} / \mathrm{L}$ vs noDCI 0.050 , IQR $0.062 \mu \mathrm{g} / \mathrm{L} ; \mathrm{p}<$ 0.001 ) within this selected group of patients (Fig. 4A). Patients developing DCI refractory to treatment and evolving into cerebral infarctions $(n=7)$ had significantly higher PCT levels compared with those not developing infarction (n = 17) $\left(\mathrm{DCI}_{\text {infarct }} 0.170, \mathrm{IQR} 0.235 \mu \mathrm{g} / \mathrm{L}\right.$ vs $\mathrm{DCI}_{\text {no-infarct }}$ 0.048 , IQR $0.063 \mu \mathrm{g} / \mathrm{L} ; \mathrm{p}=0.002$ ) (Fig. 4B).

When analyzing PCT data around the first DCI event in the subgroup of infection-free patients, it becomes clear that pre-DCI levels plateau before DCI occurrence $(0.105$, IQR $0.114 \mu \mathrm{g} / \mathrm{L})$. The 3 days preceding DCI $\left(\mathrm{d}_{-1}\right.$ to $\left.\mathrm{d}_{-3}\right)$ 

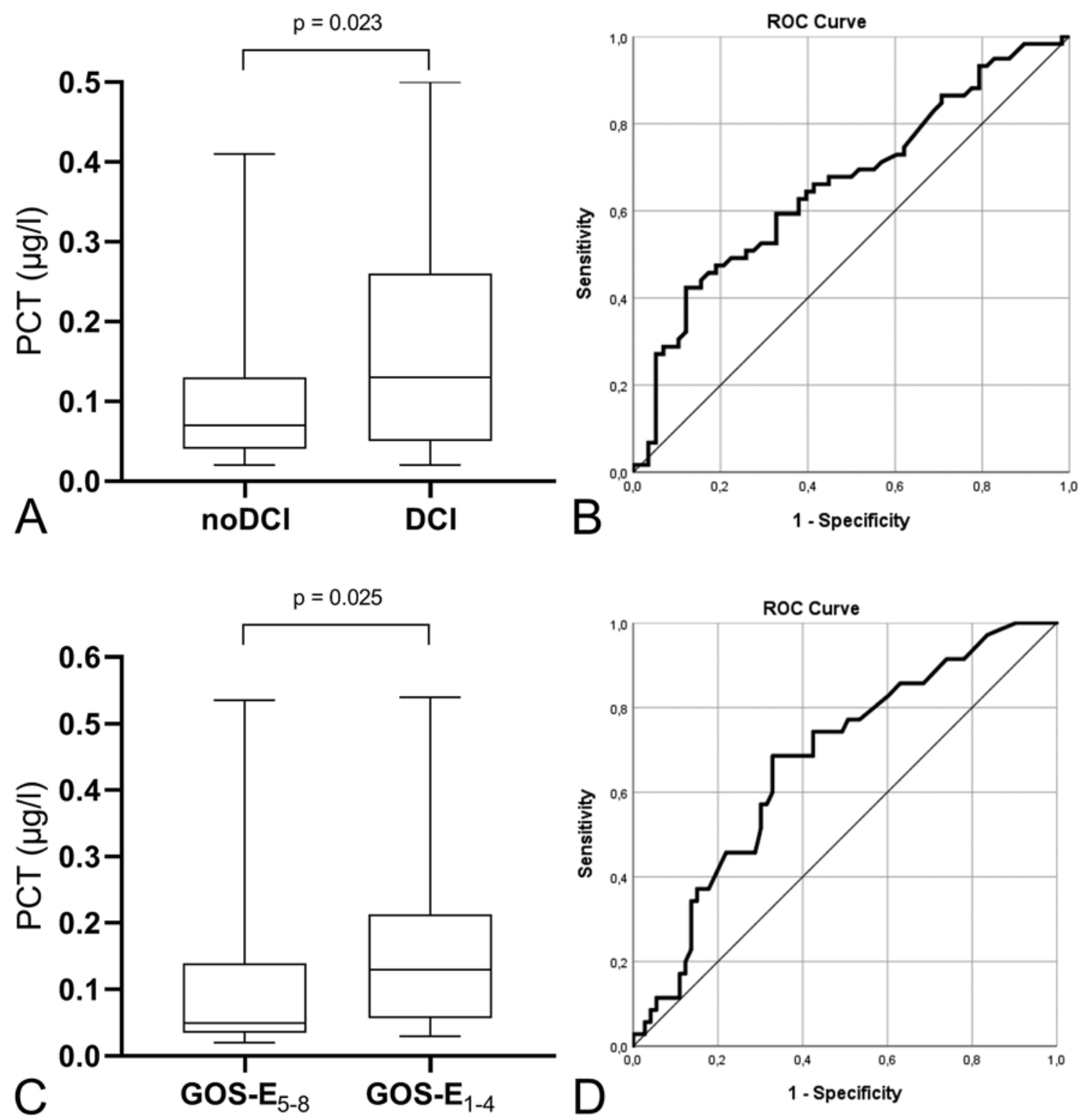

FIG. 3. Predictive value of early PCT. A: The median PCT levels of patients developing $D C l(n=64)$ in the early phase $\left(d_{0-2}\right)$ were higher compared with patients who never developed DCI $(n=68)(0.070, I Q R 0.080 \mu \mathrm{g} / \mathrm{L}$ vs $0.125, \mathrm{IQR} 0.215 \mu \mathrm{g} / \mathrm{L} ; p=0.023)$. B: ROC curve analysis shows an AUC of 0.661 and SE of $0.050(p=0.003)$ demonstrating a low predictive value of PCT levels in identifying DCl-prone patients. C: Comparing early PCT $\left(\mathrm{d}_{0-2}\right)$ between dichotomized groups based on outcome data, patients eventually developing unfavorable outcome presented with higher PCT levels compared with those who recovered into favorable outcome $(0.115$, IQR $0.120 \mu \mathrm{g} / \mathrm{L}$ vs 0.060 , IQR $0.104 \mu \mathrm{g} / \mathrm{L} ; p=0.025)$. D: Early PCT levels had a low predictive value on outcome with an AUC of 0.674 and SE of $0.054(p=0.003)$.

were therefore used as a baseline reference for further comparison. When DCI occurred, PCT levels rapidly increased, reaching a peak on day 2 after DCI onset $(0.263$, IQR $0.296 \mu \mathrm{g} / \mathrm{L} ; \mathrm{p}=0.011)$. Thereafter, PCT slowly declined, but it increased again on day 8 , reaching a second slightly lower peak on day $10(0.198 \mu \mathrm{g} / \mathrm{L}, \mathrm{IQR} 0.114 \mu \mathrm{g} / \mathrm{L}$; $\mathrm{p}=0.003)$ (Fig. 4C).

\section{PCT Levels in DCl-Related Cerebral Infarction}

When comparing patients with successful DCI treatment $\left(\mathrm{DCI}_{\mathrm{no-infarction}}\right)$ and patients with DCI refractory to treatment and progressing toward cerebral infarction $\left(\mathrm{DCI}_{\text {infarction }}\right)$, different characteristics of PCT levels were observed. There was a comparable initial pre-DCI plateau with median values of 0.110 , IQR $0.014 \mu \mathrm{g} / \mathrm{L}$ in the infarction-free subgroup, and 0.064, IQR $0.008 \mu \mathrm{g} / \mathrm{L}$ in the infarction subgroup $(\mathrm{p}=0.086)$. In both groups PCT increased after DCI onset, reaching its peak at approximately day $2\left(\mathrm{DCI}_{\text {no-infarction }} 0.266\right.$, IQR $0.101 \mu \mathrm{g} / \mathrm{L}$ and $\mathrm{DCI}_{\text {infarction }} 0.254, \mathrm{IQR} 0.094 \mu \mathrm{g} / \mathrm{L}$ compared with baseline $[\mathrm{p}=0.121$ and $\mathrm{p}=0.011$, respectively $]$ ), after which levels quickly decreased again $\left(\mathrm{d}_{3-7}\right)$. In those patients with 

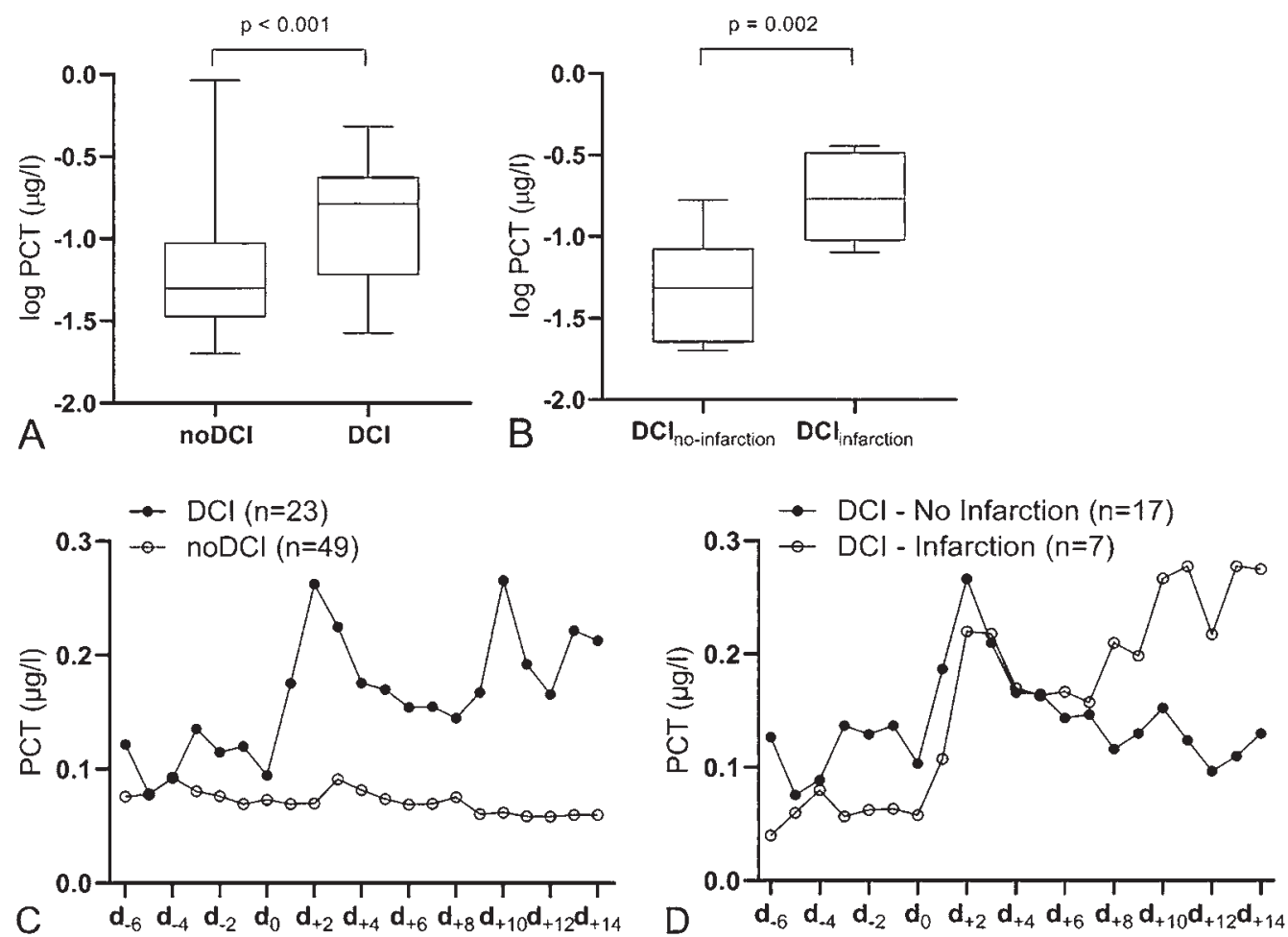

FIG. 4. Subgroup comparisons of PCT levels. For every subgroup, an appropriate time frame was identified. Because absolute PCT values are positively skewed, data were logarithmically transformed before plotting. A: PCT values of the day of DCl occurrence and the 2 following days were used to compare the $\mathrm{DCl}(n=23)$ and noDCl $(n=49)$ subgroups. In the noDCl subgroup, PCT levels from $d_{6-8}$ posthemorrhage were used (mean $\mathrm{DCl}$ occurrence at $\mathrm{d}_{6}$ in this cohort). B: Pooled PCT data on the day of $\mathrm{DCl}$ occurrence and the 2 following days were used to compare the infarction $(n=7)$ and no-infarction $(n=17)$ subgroups. The time course of PCT (in $\mu \mathrm{g} / \mathrm{L}$ ) was plotted around the $\mathrm{DCl}$ event. For the $\mathrm{DCl}$ group, $\mathrm{d}_{0}$ is the day of the first $\mathrm{DCl}$ event. For the noDCl group, data were synchronized with the $\mathrm{DCl}$ group by using day 6 as time point $\mathrm{d}_{0}(6$ days postictus was the mean lag of $\mathrm{DCl}$ occurrence in the DCl group). C: The median PCT levels in all patients who developed $\mathrm{DCl}(\mathrm{n}=23)$ versus patients who never developed $\mathrm{DCl}(n=49)$. $\mathrm{d}_{0}$ is the day of $\mathrm{DCl}$ development. D: Comparing PCT curves between patients developing DCl-related infarction $(n=7)$ and patients without infarction $(n=17)$. Curves were compared with a repeated-measures ANOVA $(F[1.0,1.0]=$ $6.954, p=0.231$ ). Due to the wide dispersion of data and for clearer illustration, no measure of dispersion was added to the figure.

successful treatment, PCT levels declined continuously, reaching baseline values again at approximately day 12 . In the case of refractory treatment, PCT levels started increasing again in the infarction subgroup at approximately day 8 , to reach an even higher peak on day 14 after DCI (0.278, IQR $0.028 \mu \mathrm{g} / \mathrm{L} ; \mathrm{p}<0.001$ ) (Fig. 4D).

\section{Discussion}

In previous studies, the role of multiple inflammatory biomarkers during the time course after aSAH and their correlation with DCI occurrence were investigated, ${ }^{11,14,27}$ failing, however, to identify a reliable biomarker for the occurrence of DCI or cerebral infarction.

In the context of an underlying inflammatory process during and after aSAH, we evaluated the course of PCT levels after aSAH in this prospective observational study. In healthy individuals, PCT is present in undetectable levels. However, concentrations increase rapidly in the case of bacterial infections and decrease steadily with the initiation of antibiotic treatment. Elevated PCT levels can also be observed in conditions unrelated to infection, for example, in the context of severe trauma. ${ }^{16}$
Our main analysis focused on the predictive value of early PCT levels with respect to DCI occurrence and outcome. Due to the disturbing influence of nosocomial infections on PCT, the infection-free subgroup was isolated from this series of consecutive patients for specific analyses. Because PCT levels did not differ during the first 3 days after admission between patients developing or not developing nosocomial infections, all patients' data were used when analyzing PCT levels from these time points. An ROC analysis showed a predictive value of pre-DCI levels of PCT in foreseeing DCI development, and the predictive values were comparable to those calculated in this cohort for the modified Fisher scale and HH grading scale as well as the VASOGRADE risk stratification model for DCI. ${ }^{28}$ A more elaborate DCI prediction model including laboratory results such as PCT levels could assist in improving the risk stratification for DCI in patients with aSAH. We hypothesize that early PCT reflects the severity of EBI, which was also illustrated by higher PCT concentration in poor-grade patients. The contribution of EBI to DCI development has been clearly established, which can explain PCT's predictive value for DCI occurrence. ${ }^{29}$

PCT levels in infection-free patients with aSAH paral- 
leled the course of DCI development by a rapid increase, reaching peak values at approximately day 2 after DCI onset. After this first spike, levels initially decreased gradually, with the exception of a secondary increase at approximately days 11 and 13 in patients progressing to cerebral infarction.

Three previous trials examined PCT levels in patients with aSAH, focusing, among other issues, on the applicability of PCT as a sepsis marker during the first 7 days in an aSAH cohort..$^{30}$ Muroi et al. discovered that PCT levels were significantly higher in patients with more severe aSAH as measured by the World Federation of Neurosurgical Societies grading scale. ${ }^{31}$ In this prospective trial, PCT samples were collected only during the first 24 hours after hemorrhage. In our analysis, early PCT correlated with the initial clinical grade as well. In another larger data collection, PCT levels were evaluated during 14 days after hemorrhage. ${ }^{32}$ Lower PCT levels on days 6 and 8-14 correlated with favorable clinical outcome as measured by the GOS after 3 months. In this trial, there was no distinction made between patients with and those without bacterial infection, because the authors stated that there was no difference in PCT values between those groups.

As was anticipated, the exclusion of patients with infections from specific subgroup DCI analyses resulted in significantly fewer patients with poor-grade $\left(\mathrm{HH}_{\mathrm{III-V}}\right)$ aSAH in the infection-free subgroup. The better clinical condition of this subgroup is also illustrated by a lower proportion of patients requiring ventilation and an overall shorter length of ventilation in the infection-free subgroup. To isolate the effect of DCI on PCT levels, we introduced a selection bias for less severely ill patients with aSAH who were at lower risk of DCI and DCI-caused infarction (depicted in Table 1). This is an artificially created situation because, in reality, during the first days of ICU stay, it is unclear which patients will develop a nosocomial infection.

Systemic inflammatory parameters will remain hampered in their ability to detect noninfectious inflammatory processes by their lack of sensitivity. As we pointed out earlier, the underlying pathophysiological mechanism of the ongoing neuroinflammatory response after aSAH is highly complex and many processes are cross-linked. We only analyzed a single parameter; the interactions between individual systemic inflammatory parameters IL-1, IL-6, CRP, and PCT as well as leukocyte count could be of interest in future research..$^{15,31,32}$ Even more fundamentally, it is unclear whether these inflammatory cytokines and biomarkers are part of a protective or detrimental process. ${ }^{27}$

In this analysis, we did not evaluate PCT levels in CSF because CSF collection was not part of the routine data acquisition. Alons et al. demonstrated that PCT in CSF is detectible and could be a valuable marker differentiating bacterial from viral meningitis in an early stage. ${ }^{33}$ Moreover, a recent study showed that PCT can be produced within the CNS itself, by trigeminal glial cells in response to inflammation. ${ }^{34}$ It could be possible that PCT levels in CSF do not respond to systemic bacterial infections, making it a potentially more sensitive target for DCI prediction and surveillance. The ability to identify an infection-independent predictive biomarker for DCI would be a major breakthrough, avoiding expensive and labor-intensive in- vasive diagnostics or exposure to radiation during radiological examinations.

We did not take into account possible PCT changes due to aneurysm occlusion procedures, as well as other invasive procedures during the ICU stay, because their effects have proved to be negligible. ${ }^{35}$ The highest PCT value in infection-free patients with aSAH was $0.95 \mu \mathrm{g} / \mathrm{L}$ on day 7 postictus in a patient with $\mathrm{HH}$ grade III during DCI treatment with infarct demarcation later on. It is safe to say that, in general, absolute elevated PCT levels due to DCI development never reach peaks comparable to those caused by bacterial infection, eliminating the danger that starting antibiotic treatment due to DCI might have caused PCT increase.

Finally, although patients were recruited over 4 years' time, our sample size remains fairly small. Additionally, the variance of PCT data is known to be high, making the interpretation of individual values in daily practice difficult. In reality, the temporal course of relative changes might be a better measure to keep in mind when evaluating infection-free patients with aSAH who are at risk of DCI. Nonetheless, to our knowledge this is the largest prospective cohort assessing PCT in relation to DCI in patients with aSAH.

\section{Conclusions}

This observational trial shows the predictive value of PCT levels in identifying DCI-susceptible patients with aSAH and patients developing an unfavorable outcome. The clinical use in confirmation of DCI will remain limited because PCT measurements become overshadowed by changes caused by nosocomial infections. In infectionfree patients, PCT levels are indicative of DCI treatment failure and could be used as a readily available biomarker for DCI treatment surveillance.

\section{References}

1. Dijkland SA, Jaja BNR, van der Jagt M, et al. Between-center and between-country differences in outcome after aneurysmal subarachnoid hemorrhage in the Subarachnoid Hemorrhage International Trialists (SAHIT) repository. J Neurosurg. 2020;133(4):1132-1140.

2. Pobereskin LH. Incidence and outcome of subarachnoid haemorrhage: a retrospective population based study. J Neurol Neurosurg Psychiatry. 2001;70(3):340-343.

3. Vergouwen MD, Etminan N, Ilodigwe D, Macdonald RL. Lower incidence of cerebral infarction correlates with improved functional outcome after aneurysmal subarachnoid hemorrhage. J Cereb Blood Flow Metab. 2011;31(7):15451553.

4. Terpolilli NA, Brem C, Bühler D, Plesnila N. Are we barking up the wrong vessels? Cerebral microcirculation after subarachnoid hemorrhage. Stroke. 2015;46(10):3014-3019.

5. Anzabi M, Angleys H, Aamand R, et al. Capillary flow disturbances after experimental subarachnoid hemorrhage: a contributor to delayed cerebral ischemia? Microcirculation. 2019;26(3):e12516.

6. Balbi M, Koide M, Wellman GC, Plesnila N. Inversion of neurovascular coupling after subarachnoid hemorrhage in vivo. J Cereb Blood Flow Metab. 2017;37(11):3625-3634.

7. Liu H, Dienel A, Schöller K, et al. Microvasospasms after experimental subarachnoid hemorrhage do not depend on endothelin A receptors. Stroke. 2018;49(3):693-699. 
8. Rass V, Helbok R. Early brain injury after poor-grade subarachnoid hemorrhage. Curr Neurol Neurosci Rep. 2019; 19(10):78.

9. de Oliveira Manoel AL, Macdonald RL. Neuroinflammation as a target for intervention in subarachnoid hemorrhage. Front Neurol. 2018;9:292.

10. McBride DW, Blackburn SL, Peeyush KT, et al. The role of thromboinflammation in delayed cerebral ischemia after subarachnoid hemorrhage. Front Neurol. 2017;8:555.

11. Chamling B, Gross S, Stoffel-Wagner B, et al. Early diagnosis of delayed cerebral ischemia: possible relevance for inflammatory biomarkers in routine clinical practice? World Neurosurg. 2017;104:152-157.

12. Juvela S, Kuhmonen J, Siironen J. C-reactive protein as predictor for poor outcome after aneurysmal subarachnoid haemorrhage. Acta Neurochir (Wien). 2012;154(3):397-404.

13. Turner CL, Budohoski K, Smith C, et al. Elevated baseline C-reactive protein as a predictor of outcome after aneurysmal subarachnoid hemorrhage: data from the Simvastatin in Aneurysmal Subarachnoid Hemorrhage (STASH) Trial. Neurosurgery. 2015;77(5):786-793.

14. Höllig A, Remmel D, Stoffel-Wagner B, et al. Association of early inflammatory parameters after subarachnoid hemorrhage with functional outcome: a prospective cohort study. Clin Neurol Neurosurg. 2015;138:177-183.

15. Sarrafzadeh A, Schlenk F, Gericke C, Vajkoczy P. Relevance of cerebral interleukin-6 after aneurysmal subarachnoid hemorrhage. Neurocrit Care. 2010;13(3):339-346.

16. Mimoz O, Benoist JF, Edouard AR, et al. Procalcitonin and C-reactive protein during the early posttraumatic systemic inflammatory response syndrome. Intensive Care Med. 1998; 24(2):185-188.

17. Chou SH, Macdonald RL, Keller E. Biospecimens and molecular and cellular biomarkers in aneurysmal subarachnoid hemorrhage studies: common data elements and standard reporting recommendations. Neurocrit Care. 2019;30(suppl 1):46-59.

18. Thompson K, Venkatesh B, Finfer S. Sepsis and septic shock: current approaches to management. Intern Med J. 2019;49(2): $160-170$.

19. Albanna W, Weiss M, Müller M, et al. Endovascular rescue therapies for refractory vasospasm after subarachnoid hemorrhage: a prospective evaluation study using multimodal, continuous event neuromonitoring. Neurosurgery. 2017;80(6): 942-949.

20. Weiss M, Conzen C, Mueller M, et al. Endovascular rescue treatment for delayed cerebral ischemia after subarachnoid hemorrhage is safe and effective. Front Neurol. 2019;10:136.

21. Steiner T, Juvela S, Unterberg A, et al. European Stroke Organization guidelines for the management of intracranial aneurysms and subarachnoid haemorrhage. Cerebrovasc Dis. 2013;35(2):93-112.

22. Hutchinson P, O'Phelan K. International multidisciplinary consensus conference on multimodality monitoring: cerebral metabolism. Neurocrit Care. 2014;21(suppl 2):S148-S158.

23. Le Roux P, Menon DK, Citerio G, et al. The International Multidisciplinary Consensus Conference on Multimodality Monitoring in Neurocritical Care: evidentiary tables: a statement for healthcare professionals from the Neurocritical Care Society and the European Society of Intensive Care Medicine. Neurocrit Care. 2014;21(suppl 2):S297-S361.

24. Vergouwen MD, Vermeulen M, van Gijn J, et al. Definition of delayed cerebral ischemia after aneurysmal subarachnoid hemorrhage as an outcome event in clinical trials and observational studies: proposal of a multidisciplinary research group. Stroke. 2010;41(10):2391-2395.

25. Singer M, Deutschman CS, Seymour CW, et al. The third international consensus definitions for sepsis and septic shock (Sepsis-3). JAMA. 2016;315(8):801-810.
26. Jennett B, Snoek J, Bond MR, Brooks N. Disability after severe head injury: observations on the use of the Glasgow Outcome Scale. J Neurol Neurosurg Psychiatry. 1981;44(4): 285-293.

27. Höllig A, Stoffel-Wagner B, Clusmann H, et al. Time courses of inflammatory markers after aneurysmal subarachnoid hemorrhage and their possible relevance for future studies. Front Neurol. 2017;8:694.

28. de Oliveira Manoel AL, Jaja BN, Germans MR, et al. The VASOGRADE: a simple grading scale for prediction of delayed cerebral ischemia after subarachnoid hemorrhage. Stroke. 2015;46(7):1826-1831.

29. Ahn SH, Savarraj JP, Pervez M, et al. The subarachnoid hemorrhage early brain edema score predicts delayed cerebral ischemia and clinical outcomes. Neurosurgery. 2018;83(1): 137-145.

30. O'Connor E, Venkatesh B, Mashongonyika C, et al. Serum procalcitonin and $\mathrm{C}$-reactive protein as markers of sepsis and outcome in patients with neurotrauma and subarachnoid haemorrhage. Anaesth Intensive Care. 2004;32(4):465-470.

31. Muroi C, Lemb JB, Hugelshofer M, et al. Early systemic procalcitonin levels in patients with aneurysmal subarachnoid hemorrhage. Neurocrit Care. 2014;21(1):73-77.

32. Muroi C, Hugelshofer M, Seule M, et al. Correlation among systemic inflammatory parameter, occurrence of delayed neurological deficits, and outcome after aneurysmal subarachnoid hemorrhage. Neurosurgery. 2013;72(3):367-375.

33. Alons IM, Verheul RJ, Kuipers I, et al. Procalcitonin in cerebrospinal fluid in meningitis: a prospective diagnostic study. Brain Behav. 2016;6(11):e00545.

34. Raddant AC, Russo AF. Reactive oxygen species induce procalcitonin expression in trigeminal ganglia glia. Headache. 2014;54(3):472-484.

35. Meisner M, Tschaikowsky K, Hutzler A, et al. Postoperative plasma concentrations of procalcitonin after different types of surgery. Intensive Care Med. 1998;24(7):680-684.

\section{Disclosures}

The authors report no conflict of interest concerning the materials or methods used in this study or the findings specified in this paper.

\section{Author Contributions}

Conception and design: Albanna, Veldeman, Lepore. Acquisition of data: Albanna, Veldeman, Lepore. Analysis and interpretation of data: Albanna, Veldeman, Lepore, Höllig, Stoppe, Schubert. Drafting the article: Veldeman, Schubert. Critically revising the article: Albanna, Lepore, Höllig, Clusmann, Stoppe, Schubert. Reviewed submitted version of manuscript: all authors. Approved the final version of the manuscript on behalf of all authors: Albanna. Statistical analysis: Albanna, Veldeman. Administrative/ technical/material support: Albanna, Lepore. Study supervision: Albanna, Höllig, Clusmann, Stoppe, Schubert.

\section{Supplemental Information}

\section{Online-Only Content}

Supplemental material is available with the online version of the article.

Supplementary Table 1. https://thejns.org/doi/suppl/10.3171/ 2020.5.JNS201337.

\section{Correspondence}

Walid Albanna: RWTH Aachen University Hospital, Aachen, Germany.walbanna@ukaachen.de. 